\title{
Management of early mobilization in intensive care units: a multicenter cross-sectional study ${ }^{\dagger}$
}

Yan-Ping Zhua, ${ }^{a, t+}$ Li-Xia Xia ${ }^{b,+\dagger}$, Guo-Hong Lic,*

aIntensive Care Units, Zhongda Hospital, Southeast University, Nanjing, Jiangsu 210009, China

${ }^{b}$ Department of Nursing, Jiangsu Provincial Hospital, Nanjing, Jiangsu 210029, China

'Department of Nursing, Zhongda Hospital, Southeast University, Nanjing, Jiangsu 210009, China

Received: 16 November 2017; Accepted: 7 February 2018; Published: 20 December 2018

Abstract: Objective: The aim of this study was to assess the management of early mobilization (EM) in Chinese intensive care units (ICUs). Methods: This survey used a cross-sectional, observational design. A total of 65 tertiary and secondary hospitals were enrolled by convenience sampling and investigated using self-designed questionnaires.

Results: We identified 69 ICUs in Jiangsu, China (response rate: $94.2 \%) .74 .2 \%(1,004 / 1,353)$ of the nurses and nursing managers from 65 ICUs reported mobility practice. For the mobility level, 98.1\% (1,327) reported use of in-bed exercise, $5.7 \%(77)$ sitting on a side of bed, $21.7 \%$ (294) transfer to chair, and $2.4 \%$ (33) walking. The most frequently reported barriers to early mobility were unplanned extubation, nursing resource, and absence of physical therapist. Nurses' educational backgrounds, nursing experience, the lack of nursing resources, absence of physician, and the weakness of patient were the factors that influenced ICU early rehabilitation $(P<0.01)$.

Conclusions: Although implementation rates for EM in critically ill patients are high, the activity level is generally poor in most of the involved ICUs.

Keywords: early mobilization $\bullet$ critical care $\bullet$ critically ill patients $\bullet$ management $\bullet$ clinical practice

(c) Shanxi Medical Periodical Press.

\section{Introduction}

Traditional standards of practice do not ambulate intensive care unit (ICU) patients because of their critical illness and adverse events. ${ }^{1-4}$ However, recent studies show that immobility in the ICUs plays an important role in the development of ICU-acquired weakness, ICU delirium, higher mortality rates, and increased cost of treatment. ${ }^{5-7}$ To improve outcomes of critically ill patients, 2013 ICU PAD guidelines, the European Respiratory Society and European Society of Intensive

${ }^{\dagger}$ The authors would like to thank Pan C and Wu C for their cooperation in this study. We also thank $\mathrm{He} L$ for administrative support.

${ }^{+\dagger}$ Yan-Ping Zhu and Li-Xia Xia contributed equally to this study.

* Corresponding author.

E-mail: njtxq512@163.com (G.-H. Li).

Ә Open Access. (C) 2018 Yan-Ping Zhu et al., published by Sciendo. Attribution NonCommercial-NoDerivatives 4.0 License.
Care Medicine suggests early mobilization (EM) in ICU, which includes active and passive, in-bed and out-bed exercises. ${ }^{8,9}$ Several single and multicenter research studies, systematic reviews, and a meta-analysis have recommended that implementation of early mobility can benefit functional status and survival. ${ }^{10-20}$

Whether Chinese ICUs follow EM is unknown. Disseminating guidelines generally is insufficient to change clinical practice. ${ }^{21}$ Country-level efforts to promote the EM guideline is discrepancy in most of China, where insufficient financial and supporting disciplinary may limit the ability to implement potentially early mobility in critical care services. To make the problem even worse, the evidence-based intervention strategies identified so far lack enough resource to promote early mobility as a routine care for critically ill patients. ${ }^{16-22}$ Moreover, most 
of the previous studies were carried out in developed countries, where resource and health-care system were quite different from that in China. ${ }^{23}$

Based on these considerations, we evaluated the implementation of EM in Chinese ICUs and hospitals. We also assessed perceived barriers to implementation of EM within the department and the factors associated independently with current practice.

\section{Materials and methods}

\subsection{Study design}

The Management of Early Mobilization in Chinese Intensive Care Units study, a large multicenter research of Chinese ICUs, represents a meaningful step toward collaborative Chinese intensive care research on EM. An annual committee of nurses and nursing managers from various Chinese ICUs, formed in May 2017, invited nursing managers representing ICUs in their hospitals to participate through telephone contact, emails, and express delivery. The participants were encouraged to include as many as possible to minimize selection bias. Participation in the research was voluntary and unfunded.

Participating ICUs had at least six beds in each, they could be medical, surgical, or mixed, except for pediatric ICUs, according to "Hospital grade management standards defined by National Health and Family Planning Commission" in Jiangsu, China. We enrolled all nurses of recruited ICUs in their centers to participate through representing nursing managers. There was no sample size calculation in this survey.

\subsection{Data collection}

In this research, EM was defined as any activity beyond range of motion performed by a care provider (nursing or physical therapist) for ICU patients, including active and passive, in-bed and out-bed exercises (positioning, bed mobility, sitting on side of bed, transfer to chair, walking, etc.). ${ }^{9,15,16}$ Our survey was developed through an iterative process involving the framework in the study by Cabana et al., ${ }^{21}$ literature review, and critical care nurses. ${ }^{24,25} \mathrm{~A}$ preliminary survey was carried out in several ICU nurses with critical care experience in a university hospital to access for feasibility. Modifications based on the nurses' suggestions resulted in the final version of the survey. The survey was divided into two parts.

Part 1 addressed organizational characteristics, including type of hospital (government university and non-university), specialty of ICUs (medical, surgical, or mixed), number of beds, bed-to-nurse ratio in ICU, number of intensive nurses, nurse age, sex, education, nursing, and critical care experience.

Part 2 of the survey included process parameters as follows: EM implementation, especially for patients on mechanical ventilation (MV) or continuous renal replacement therapy (CRRT) or extracorporeal membrane oxygenation (ECMO). The part also recorded perceived barriers and knowledge regarding current practice in the ICUs.

This survey was conducted between July 1 to July 14, 2017. Data were recorded by one researcher and then independently checked by two other researchers. We checked the recorded data for statistical outliers and contacted the representing nursing managers for interpretation.

\subsection{Statistical analyses}

Categorical variables are given as number (percentage for implementation of EM), normally distributed numerical variables as mean (standard deviation), and other numerical variables as median (interquartile range). Categorical variables were analyzed with Chi-square test or Fisher's exact test.

In assessing implementation of EM, nurses who practiced all or one of the EM protocols were deemed implementation. 9,15,16 To identify the independent factors associated with implementation, we compared binary variable logistic regression analysis using models that included the implementation of EM protocols and all the variables collected for structure and process characteristics. Model fit was assessed with the Hosmer-Lemeshow goodness of fit test. SPSS 18.0 (SPSS, Inc, Chicago, IL, USA) was used for statistical analysis. A $P$-value of $<0.05$ is considered to be significant.

\section{Results}

Of the 69 ICUs contacted, 65 ICUs completed surveys form, an overall response rate of $94.2 \%$ was achieved in this study, enrolling 1,353 nurses, which accounted for $21 \%$ of all university and non-university hospitals during the study period in Jiangsu province, China.

\subsection{EM practice}

Most of the participating hospitals were university hospitals (85\%), which had $11-20$ ICU beds (46.2\%) and bed-to-nurse ratio in ICU between 1 and 2 (55.4\%). Overall, $74.2 \%(1,004 / 1,353)$ of patients reported EM practice, with $74.6 \%(894 / 1,199)$ of the university hospitals and $71.4 \%(110 / 154)$ of the non-university hospitals, respectively. The difference was not significant, 
although university hospitals were associated with a higher implementation of EM (each with a $P$ value of $>0.05$ ). The five critical attending nursing factors that were related to the implementation of EM are as follows: nurses' age less than 25 years, male gender, technical secondary education, 5-10 years of nursing experience, and critical attending experience less than 5 years, which reported higher EM rates in ICUs (each with a $P$ value of $<0.05)$. We identified six patient-specific treatments as follows: non-invasive mechanical ventilation (NIPPV), intubation, tracheotomy, CRRT-jugular vein catheter, CRRT-femoral vein catheter, and ECMO, which were associated with EM implementation in ICU. EM was less likely to be practiced in ECMO patients $(P=0.000)$ (Table 1).

For the EM level, $98.1 \%(1,327)$ reported use of inbed exercise, $5.7 \%$ (77) sitting on side of bed, $21.7 \%$ (294) transfer to chair, and $2.4 \%$ (33) walking. In university hospitals, $99.4 \%$ reported bed mobility training, $6.0 \%$ sitting on side of bed, $23.3 \%$ transfer to chair, $2.7 \%$ walking, whereas $94.1 \%, 3.3 \%, 11.2 \%, 1.3 \%$, respectively, in non-university hospitals (Figure 1). For patients on MV or CRRT or ECMO, $72.5 \%$ of the nurses reported use of bed mobility training, $8.9 \%$ sitting on side of bed, $13.2 \%$ no experience, whereas transfer to chair and walking only $3.4 \%$ and $2.0 \%$, respectively (Figure 2).

\subsection{Variables independently associated with EM implementation}

On logistic regression analysis, with models that included all variables in Table 1 and Figure 3, factors independently associated with EM implementation included nurses' educational backgrounds (OR, 1.39; 95\% Cl, 1.06-1.82; $P<0.05$ ), nursing experience (OR, $0.91 ; 95 \% \mathrm{Cl}, 0.85-0.99 ; P<0.05)$, the lack of nursing resources (OR, 2.32; 95\% Cl, 1.20-4.49; $P<0.05)$, absence of physician (OR, 5.76; 95\% Cl, 1.11-29.85; $P<0.05$ ), and the weakness of patients (OR, 0.06; 95\% $\mathrm{Cl}, 0.02-0.27 ; P<0.01$ ) (Table 2). There was no interaction between these targets, and the logistic regression models fit well.

\subsection{Barriers to EM implementation}

The most frequently reported barriers to EM were unplanned extubation and lack of nursing resources (Figure 3). Most nurses indicated knowledge regarding EM; however, the agreement to transfer to chair and walking was only $69.6 \%$ (940) and 63.9\% (863), respectively.

\begin{tabular}{|c|c|c|c|}
\hline \multirow[t]{2}{*}{ Characteristics } & \multirow[t]{2}{*}{ No (\%) } & \multicolumn{2}{|c|}{$\begin{array}{l}\text { Implementation o } \\
\text { early mobilization }\end{array}$} \\
\hline & & $\%$ & $P$ \\
\hline \multicolumn{4}{|l|}{ Hospital } \\
\hline University hospital & $56(85.0)$ & 74.6 & \multirow{2}{*}{0.372} \\
\hline Non-university hospital & $9(15.0)$ & 71.4 & \\
\hline \multicolumn{4}{|l|}{ No of ICU beds } \\
\hline $1-10$ & $16(24.6)$ & 47.1 & \multirow{3}{*}{0.053} \\
\hline $11-20$ & $30(46.2)$ & 38.7 & \\
\hline$\geq 21$ & $19(29.2)$ & 73.1 & \\
\hline \multicolumn{4}{|l|}{ Bed-to-nurse ratio in ICU } \\
\hline 1 bed: $\geq 3$ nurses & $2(3.1)$ & 50.0 & \multirow{3}{*}{0.861} \\
\hline 1 bed: 2 nurses & $27(41.5)$ & 55.6 & \\
\hline$\geq 1$ bed: 1 nurse & $36(55.4)$ & 48.6 & \\
\hline \multicolumn{4}{|l|}{ Nurse age (years) } \\
\hline$\leq 25$ & $329(24.3)$ & 75.8 & \multirow{3}{*}{0.000} \\
\hline $26-40$ & $962(71.1)$ & 68.6 & \\
\hline $41-55$ & $62(4.6)$ & 51.6 & \\
\hline \multicolumn{4}{|l|}{ Nurse sex } \\
\hline Male & $147(10.9)$ & 78.2 & \multirow{2}{*}{0.000} \\
\hline Female & $1,206(89.1)$ & 72.1 & \\
\hline \multicolumn{4}{|l|}{ Education } \\
\hline Technical secondary education & $12(0.9)$ & 92.9 & \multirow{4}{*}{0.000} \\
\hline Community college & $490(36.2)$ & 73.0 & \\
\hline Undergraduate & $840(62.1)$ & 67.4 & \\
\hline Postgraduate & $11(0.8)$ & 63.6 & \\
\hline \multicolumn{4}{|l|}{ Nursing experience (years) } \\
\hline$<5$ & $669(49.4)$ & 69.7 & \multirow{4}{*}{0.000} \\
\hline $5-10$ & $438(32.4)$ & 73.5 & \\
\hline $11-20$ & $191(14.1)$ & 54.7 & \\
\hline$>20$ & $55(4.1)$ & 50.9 & \\
\hline \multicolumn{4}{|l|}{ Critical attending experience (years) } \\
\hline$<5$ & $850(62.8)$ & 73.4 & \multirow{4}{*}{0.000} \\
\hline $5-10$ & $375(27.7)$ & 67.5 & \\
\hline $11-20$ & $119(8.8)$ & 50.4 & \\
\hline$>20$ & $9(0.7)$ & 55.6 & \\
\hline \multicolumn{4}{|l|}{ Patient-specific treatments } \\
\hline NIPPV & 1,353 (19.0) & 98.9 & \multirow{6}{*}{0.000} \\
\hline Intubation & $1,353(19.0)$ & 98.4 & \\
\hline Tracheotomy & $1,353(19.0)$ & 97.9 & \\
\hline CRRT-jugular vein catheter & $1,202(16.9)$ & 85.5 & \\
\hline CRRT-femoral vein catheter & $1,197(16.8)$ & 85.0 & \\
\hline ECMO & $653(9.2)$ & 52.0 & \\
\hline
\end{tabular}

Table 1. Baseline organizational characteristics and patient-specific treatments with the implementation of early mobilization in ICU.

Notes: ICU, intensive care unit; NIPPV, noninvasive mechanical ventilation; CRRT, continuous renal replacement therapy; ECMO, extracorporeal membrane oxygenation. 


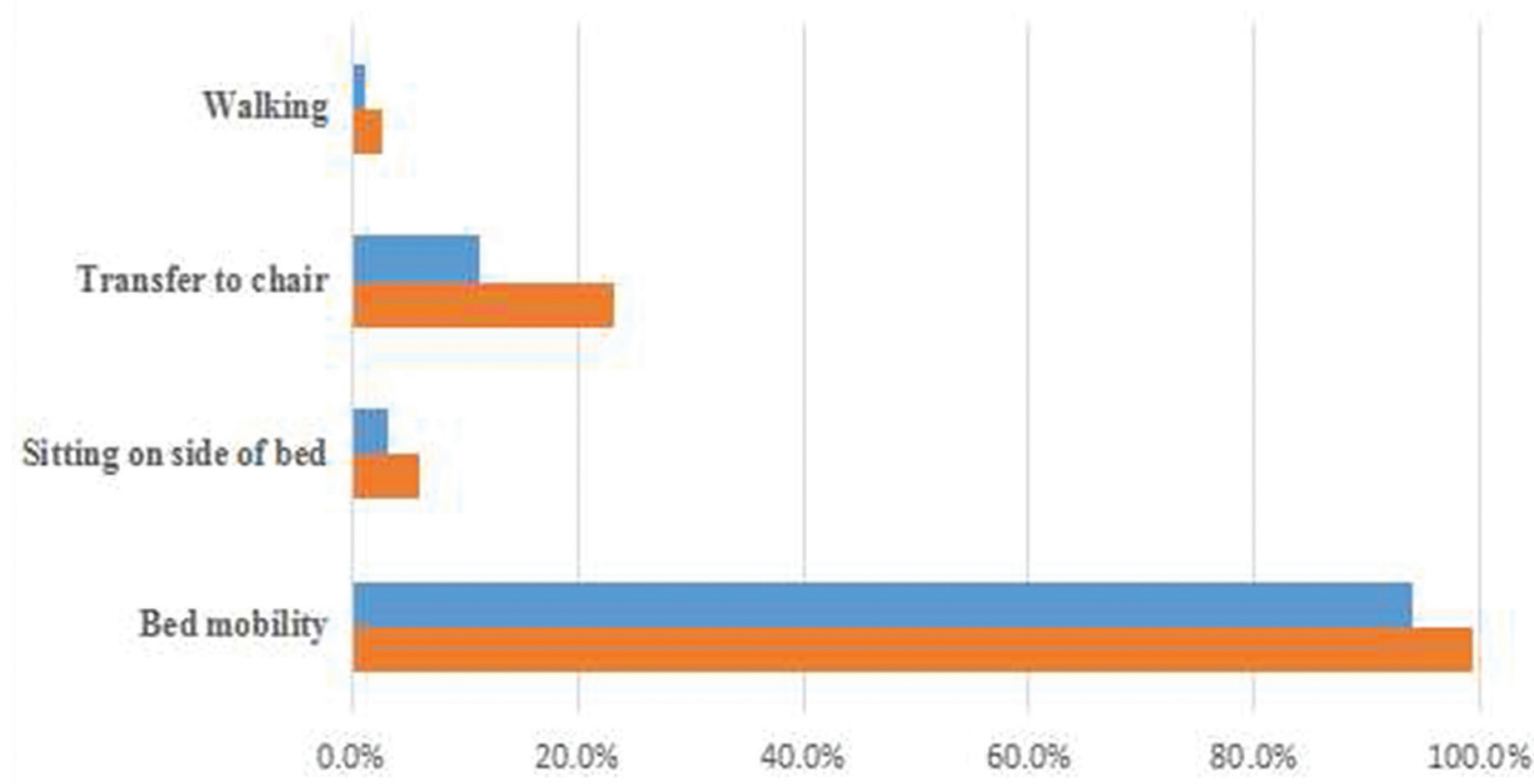

ENon-miversity hospitals $\mathbf{m}$ University hospitals

Figure 1. EM level compared in university and non-university hospitals $(n=1,353)$ Abbreviation: EM, early mobility.

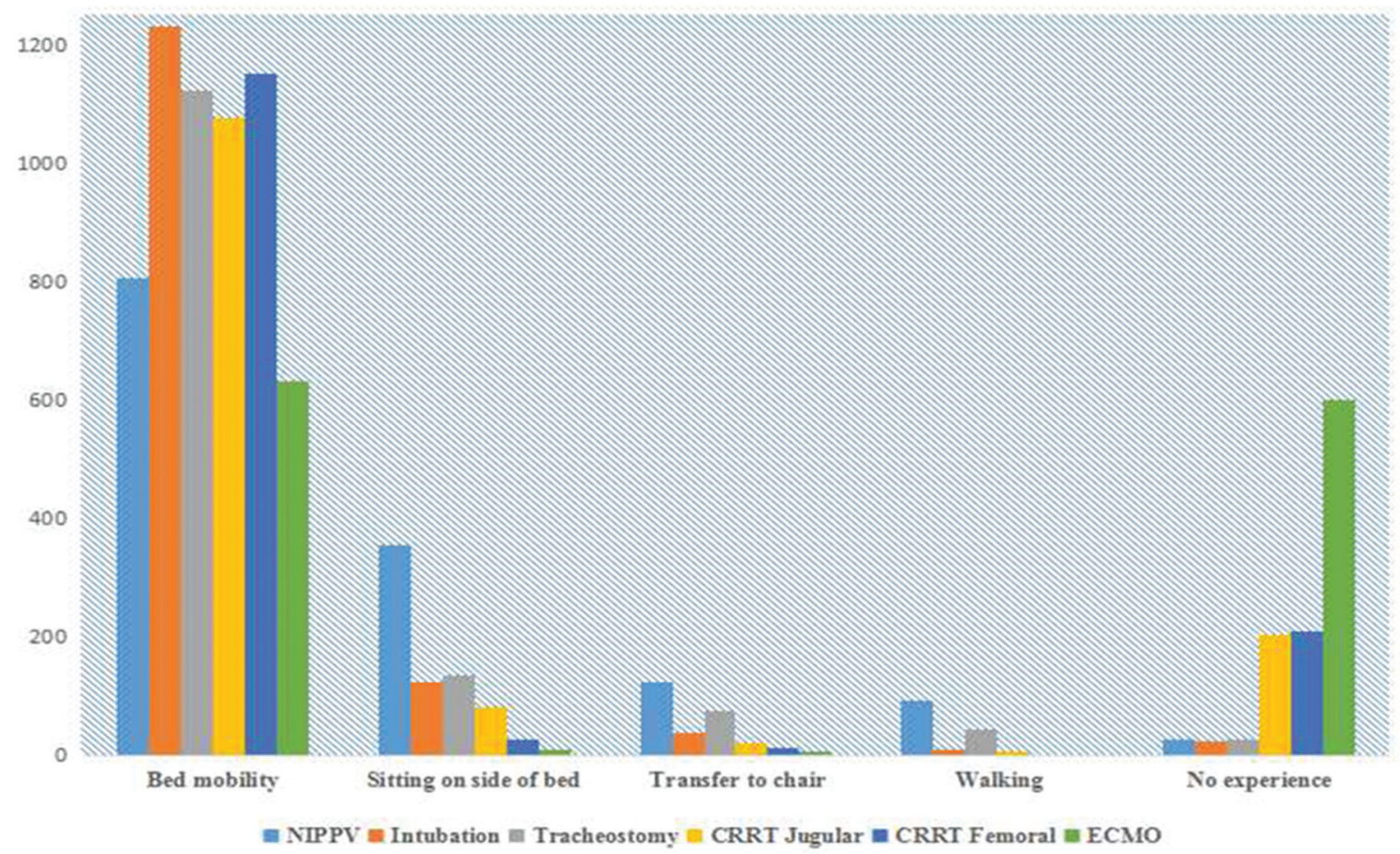

Figure 2. EM level during patient-specific treatments $(n=1,353)$.

Abbreviations: EM, early mobility; NIPPV, noninvasive mechanical ventilation; CRRT, continuous renal replacement therapy; ECMO, extracorporea membrane oxygenation. 


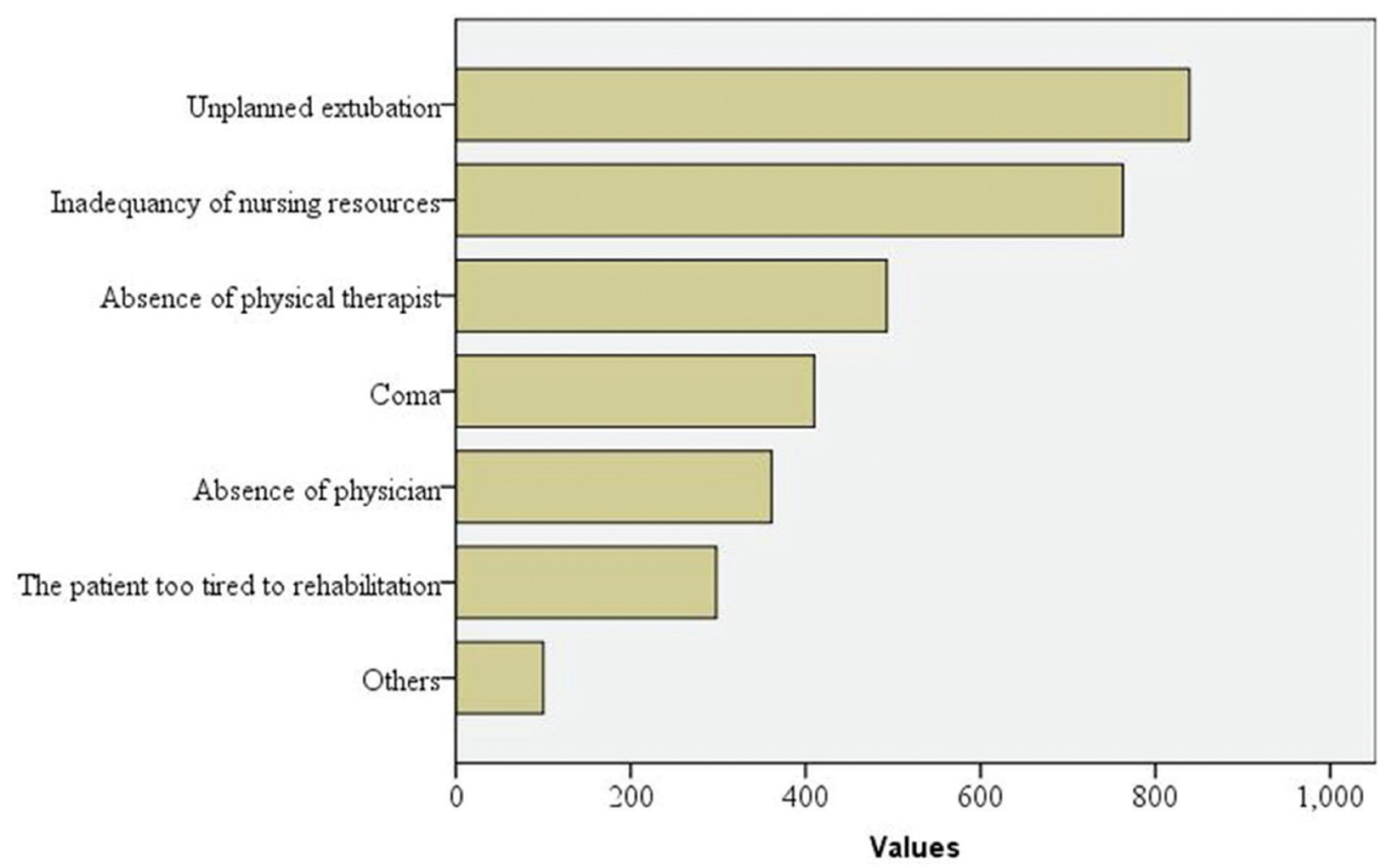

Figure 3. Nursing reported barriers to early mobility $(n=1,353)$.

\begin{tabular}{lcc}
\hline Variables & Adjusted odds ratio (95\% Cl) & $P$ \\
\hline \hline $\begin{array}{l}\text { Nurses' educational } \\
\text { backgrounds }\end{array}$ & $1.389(1.058-1.823)$ & 0.018 \\
Nursing experience & $0.914(0.846-0.987)$ & 0.022 \\
Lack of nursing resources & $2.321(1.200-4.488)$ & 0.012 \\
Absence of physician & $5.763(1.113-29.854)$ & 0.037 \\
The weakness of patients & $0.063(0.015-0.264)$ & $<0.001$ \\
\hline
\end{tabular}

Table 2. Variables independently associated with early mobility implementation on logistic regression analysis in the study of Chinese intensive care units.

Note: For models assessing nurses' educational backgrounds, nursing experience, lack of nursing resources, the weakness of patients, with the Hosmer-Lemeshow test for goodness of fit test, $R^{2}=81.010, d f=18$, and $P=0.000$.

\section{Discussion}

\subsection{Principle findings}

In the Chinese centers studied, the implementation rate for EM was $74.2 \%$. Implementation rate was considerably higher, but still incomplete, for which the most reported reasons were bed mobility training and positioning. Both nurses and nursing managers identified unplanned extubation, lack of nursing resources, and absence of physical therapist as most important barriers to EM. Nursing knowledge regarding EM in ICUs still lacks.

\subsection{Comparisons with other studies and implications}

Overall implementation of EM (74.2\%) was higher in this study than in other multicenter research studies. ${ }^{26}$ Recent implementation rates for EM were $45 \%$ in USA, ${ }^{27}$ $71 \%$ in Canada, ${ }^{28}$ and $54 \%$ in Australia. ${ }^{14}$ In this study, $74.6 \%$ of the university hospitals and $71.4 \%$ of the nonuniversity hospitals reported EM practice $(P>0.05)$.

As for the EM level, 98.1\% reported use of bed mobility training, $5.7 \%$ sitting on side of bed, $21.7 \%$ transfer to chair, and $2.4 \%$ walking. We further analyzed organizational factors and six patient-specific treatments with the EM level. For patients on MV or CRRT or ECMO, transferring to chair and walking were only $3.4 \%$ and $2.0 \%$, respectively. Similarly, a study of 116 German ICUs identified that only $6 \%$ of the patients with endotracheal tube were sitting and only $0.2 \%$ had stood out of bed or walked. ${ }^{10}$ The study of 38 ICUs in Australia and New Zealand had no patients who stood, sat out of bed, or started walking during MV. ${ }^{29}$ Dean and Ross ${ }^{30}$ found that even high-intensity exercises done in bed do not counteract the adverse effects of bed rest, recommending 
that upright positioning should be included in exercise plan of care. Taken together, these data showed that under the circumstances, the mainly achieved level of EM was in-bed exercise and more should be done to improve implementation of EM in ICUs.

In this study, it was found that hospital rank, ICU beds, and bed-to-nurse ratio in ICU are not associated with implementation rates for EM (each with a $P$ value of $>0.05$ ). First, this could be because the low level of EM practice, especially for patients on MV or CRRT or ECMO, within $72.5 \%$ reported use of in-bed exercise. Second, in this study, the average bed-to-nurse ratio in ICUs is close to $1: 2$, which is in accordance with the status of nursing resource distribution in Chinese ICUs, approaching to the guidelines (1:2.5-3) for the evaluation of hospital management in the National Health and Family Planning Commission 2008. ${ }^{31}$ In our logistic regression analysis, the independent predictors of EM implementation were nurses' educational backgrounds, years of nursing experience, the lack of nursing resources, absence of physician, and the weakness of patients (Table 2). Similarly, a recent systematic review revealed that factors associated with mobility practice included restrictive parameters, personnel resources, attitude, and perceptions. ${ }^{32}$

EM for critically ill patients is becoming an established, evidence-based practice in the ICU. ${ }^{8-13,33-37}$ Feasibility, safety, and efficiency are confirmed..$^{37}$ However, implementing guideline into practice remains challenging. What about the whole standard and progressing EM? And how to practice?

Jason et al ${ }^{38}$ suggested that barriers to adherence to the clinical practice guideline could be categorized into three areas as follows: knowledge, attitude, and behavior. We found that nurses aged less than 25 years, male, with technical secondary education, 5-10 years of nursing experience, and less than 5 years of nursing and critical care experience were more likely to practice EM (each with a $P$ value of $<0.05$ ). Most nurses indicated knowledge regarding EM; however, only $63.9 \%$ reported agreement to walking and $69.6 \%$ transferring to chair. For patient on MV or CRRT or ECMO, the out-of-bed exercise (walking and transferring to chair) accounted for $5.4 \%$. Similar research studies show that nursing knowledge and skill in achieving EM are essential. ${ }^{9}$ Implementation of a program ${ }^{39-47}$ and education of staff ${ }^{39,46}$ are important factors in EM for critically ill patients in ICU. Doherty et $\mathrm{al}^{48}$ and Garzo et $\mathrm{al}^{49}$ suggested that better definitions of care roles may allow for more targeted surveys of attitudes and behaviors that may improve EM practice.

Safety and quality of care is an eternal topic. Most nurses reported their knowledge regarding safety concern. Our nursing respondents indicated unplanned extubation as the first barrier to EM and lack of nursing resources as the second barrier, whereas nursing managers reported lack of nursing resources as the primary barrier and unplanned extubation as the secondary barrier. This could be because of leadership's systematic view of management. Nevertheless, both nurses and nursing managers identified unplanned extubation and lack of nursing resources as primary barriers to EM. To our relief, safety is confirmed. ${ }^{16,37,45,50-52}$ On the basis of security awareness, EM will be a better practice. In addition, EM practice is affected by the availability of resources, such as nursing staffing resources, inadequate teamwork, ${ }^{53-55}$ and protocols. ${ }^{56,57}$ Similar research studies found that the time and personnel necessary to mobility could be a deterrent to EM. ${ }^{1,28,30,58}$ Some research studies have attempted to overcome this difficulty by prioritizing daily care routines to EM, simplified guidelines, ${ }^{58-60}$ and creating mobility teams. ${ }^{14}$ Bakhru et $\mathrm{al}^{27}$ separated barriers into institutional barriers, patient barriers, and health-care providers barriers, suggesting a better understanding of potential benefits of EM and concerns about patients' need of education and guidance on patient mobility techniques.

\subsection{Study strengths and limitations}

The IOEMCICUS study, a large multicenter study of Chinese ICUs, represents a meaningful step toward collaborative Chinese intensive care research on EM. To make the study more representative, we included a large number of participating centers, though because of a lack of funding this resulted in a need for compromise. This study is subject to selection bias and might not fully reflect intensive care through China. ICUs with less than six beds were excluded, many of the Jiangsu, Chinese Least Developed Cities did not participate, and university hospitals accounted for $85 \%$ of the centers. The latter two points stand out when one considers the fact that all participating units could perform positioning, bed mobility training, sitting on side of bed, transfer to chair, and walking, part of which are relative luxury in many resource poor areas in Jiangsu, China. Importantly, the number of nurses in the study sample was not proportionate to the number of nurses of China taking part in the study. About $89 \%$ of the nurses were enrolled in 56 university hospitals and only $11 \%$ in nine non-university hospitals. While some of these university hospitals have good resources, others (such as secondary hospitals) have relatively poor conditions, and results from the hospitals would have a disproportionate effect on overall implementation rates. While section bias is likely, we note that the difference in EM implementation rates was not significant between university and nonuniversity hospitals in this study. 
A current definition of EM refers to the application of physical activity within the first 2-5 days of critical illness or injury; ${ }^{26}$ however, in our research, EM may be beyond the "window". The net effect of "the window" bias could be an overestimate of EM implementation. Other limitations include potential interactions that may exist between survey questions that may introduce response bias. Finally, although we did not systematically check data accuracy, we did design the data entry forms to disallow missing fields and contact ICU representatives for clarification of any outlying data.

In summary, taking into account selection bias including the cross-sectional design, the under-representation of the Chinese poor-equipment hospitals, and the overrepresentation of university hospitals, the net effect of the "window" bias, true implementation rates for EM, is likely to be lower than our data. We suggest that given the conditions in China, the most appropriate strategy might be to focus on organizational structure (mobility team), process structure (protocols, risk management, education, checking feedback, and improvement), and outcome structure (the efficiency of intervention program, functional outcome of patients, and the costs and benefits).

\section{Conclusions}

Although implementation of EM is high, the activity level is generally poor in most areas of China. Nursing knowledge and the specific implementation of EM in the ICU are lacking. Understanding the barriers to and facilitators of implementing the guidelines is important for

\section{References}

1. Schweickert WD, Kress JP. Implementing early mobilization interventions in mechanically ventilated patients in the ICU. Chest. 2011;140:16121617.

2. Jackson JC, Santoro MJ, Ely TM, et al. Improving patient care through the prism of psychology: application of Maslow's hierarchy to sedation, delirium, and early mobility in the intensive care unit. J Crit Care. 2014;29:438-444.

3. Pawlik A. Early mobilization in the management of critical illness. Crit Care Nurs Clin North Am. 2012;4:481-490.

4. Ross AG, Morris PE. Safety and barriers to care. Crit Care Nurse. 2010;30:S11-S13.

5. Allen C, Glasziou P, Del Mar C. Bed rest: a potentially harmful treatment needing more careful evaluation. Lancet. 1999;354:1229-1233. developing effective implementation strategies. Further studies may highlight nurse-led EM guideline practice, which substantially improves the outcomes of the critically ill patients.

\section{Contributors}

All authors participated in the design of the study. YanPing Zhu conceived the study. Yan-Ping Zhu and Li-Xia Xia performed the statistical analyses and wrote the report. Guo-Hong Li revised the text. All the authors approved the final version of the report.

\section{Competing interests}

All authors declare that we have no financial and personal relationships with other people or organizations that can inappropriately influence our work, and there are no professional or other personal interests of any nature or kind in any product, service and/or company that could be construed as influencing the position presented in, or the review of, the manuscript entitled.

\section{Data sharing}

Statistical code is available from the corresponding author at njtxq512@163.com.

\section{Conflicts of interest}

All contributing authors declare no conflicts of interest.

6. Needham DM. Mobilizing patients in the intensive care unit: Improving neuromuscular weakness and physical function. JAMA. 2008;300:1685-1690.

7. Puthucheary Z, Rawal J, Ratnayake G, Harridge $\mathrm{S}$, Montgomery $\mathrm{H}$, Hart N. Neuromuscular blockade and skeletal muscle weakness in critically ill patients: Time to rethink the evidence? Am J Respir Crit Care Med. 2012;185:911-917.

8. Barr J, Fraser GL, Puntillo K, et al. American College of Critical Care Medicine: Clinical practice guidelines for the management of pain, agitation, and delirium in adult patients in the intensive care unit. Crit Care Med. 2013;41: 263-306.

9. Gosselink R, Bott J, Johnson M, et al. Physiotherapy for adult patients with critical illness: recommendations of the European respiratory society and 
European society of intensive care medicine task force on physiotherapy for critically ill patients. Intensive Care Med. 2008;34:1188-1199.

10. Devlin JW, PohIman AS. Everybody, every day: an "awakening and breathing coordination, delirium monitoring/management, and early exercise/mobility" culture is feasible in your ICU. Crit Care Med. 2014;42:1280-1281.

11. Lewis M. Intensive care unit rehabilitation within the United Kingdom: Review. Physiotherapy. 2003;89:531-853.

12. Nydahl P, Ruhl AP, Bartoszek G, et al. Early mobilization of mechanically ventilated patients: a 1-day point-prevalence study in Germany. Crit Care Med. 2014;42:1178-1186.

13. Stiller K. Physiotherapy in intensive care: an updated systematic review. Chest. 2013;144:825-487.

14. Choi J, Tasota FJ, Hoffman LA. Mobility interventions to improve outcomes in patients undergoing prolonged mechanical ventilation: a review of the literature. Biol Res Nurs. 2008;10:21-33.

15. Calvo-Ayala E, Khan BA, Farber MO, Ely EW, Boustani MA. Interventions to improve the physical function of ICU survivors: a systematic review. Chest. 2013;144:1469-1480.

16. Kayambu G, Boots R, Paratz J. Physical therapy for the critically ill in the ICU: a systematic review and meta-analysis. Crit Care Med. 2013;41:1543-1554.

17. Li Z, Peng X, Zhu B, Zhang Y, Xi X. Active mobilization for mechanically ventilated patients: a systematic review. Arch Phys Med Rehabil. 2013;94:551-561.

18. Hodgin KE, Nordon-Craft A, McFann KK, Mealer ML, Moss M. Physical therapy utilization in intensive care units: results from a national survey. Crit Care Med. 2009;37:561-566.

19. Berney S, Harrold M, Webb S, et al. Intensive care unit mobility practices in Australia and New Zealand: A point prevalence study. Intensive Care Resurc. 2013;15:260-265.

20. Skinner E, Berney S, Warrilow S, Denehy L. Rehabilitation and exercise prescription in Australian intensive care units. Physiotherapy. 2008;94:220-229.

21. Cabana MD, Rand CS, Powe NR, et al. Why don't physicians follow clinical practice guidelines? JAMA. 1999;282:1458-1466.

22. Castro-Avila AC, Seron P, Fan E, et al. Effect of rehabilitation during intensive care stay on functional status: systematic review and meta-analysis. PLoS One. 2015;10:e0130722.

23. Du B, Xi X, Chen D, Peng J. Clinical review: Critical care medicine in mainland China. Crit Care. 2010;14:206.
24. Kelley K, Clerk B, Brown V, et al. Good practice in the conduct and reporting of survey research. Int J Qual Health Care. 2003;15:261-266.

25. Elm E, Altman D, Egger M, et al. STROBE initiative: The strengthening the reporting of observational studies in epidemiology (STROE) statement: Guidelines for reporting observational studies. Epidemiology. 2007;18:800-804.

26. Hodgson C, Berney S, Harrold M, Saxena M, Bellomo R. Clinical review: Early patient mobilization in the ICU. Crit Care. 2013;17:207.

27. Bakhru RN, Wiebe DJ, McWilliams DJ, Spuhler VJ, Schweickert WD. An environmental scan for early mobilization practice in US ICUs. Crit Care Med. 2015;43:2360-2369.

28. Rose L, Fowler RA, Fan E, et al. Prolonged mechanical ventilation in Canadian intensive care units: A national survey. J Crit Care. 2015;30:25-31.

29. Hodgson C, Bellomo R, Berney S, et al. Early mobilization and recovery in mechanically ventilated patients in the ICU: A bi-national, multi-centre, prospective cohort study. Crit Care. 2015;19:81.

30. Dean E, Ross J. Discordance between cardiopulmonary physiology and physical therapy. Chest. 1992; 101:1694-1698.

31. Chen JM, You LM, Zheng J, et al. Study on the nurse characteristics and nursing work environment in medical wards, surgical wards and intensive care units in hospitals in mainland China. Chin Nurs Manag. 2012;12:20-23 (in Chinese).

32. Cameron S, Ball I, Cepinskas G, et al. Early mobilization in the critical care unit: A review of adult and pediatric literature. J Crit Care. 2015;30:664-672.

33. Kress JP, Hall JB. ICU-acquired weakness and recovery from critical illness. $N$ Engl J Med. 2014;370:1626-1635

34. Bassett RD, Vollman KM, Brandwene L, Murray T. Integrating a multidisciplinary mobility program into intensive care practice(IMMPTP): A multi-center collaborative. Intensive Crit Care Nurs. 2011;28:88-97.

35. Brownback C, Fletcher $P$, Pierce L, Klaus S. Early mobility activities during continuous renal replacement therapy. Am J Crit Care. 2014;23:348-352.

36. Morris PE, Griffin L, Berry M, et al. Receiving early mobility during an intensive care unit admission is a predictor of improved outcomes in acute respiratory failure. Am J Med Sci. 2011;341:373-377.

37. Helene L, Sylvie A, Ruddy R, et al. Systematic review of early exercise in intensive care: A qualitative approach. Anaesth Crit Care Pain Med. 2016;35:133-149.

38. Jason P, Bin D, Yao T, et al. Management of severe sepsis in patient admitted to Asian 
intensive care unit: Prospective cohort study. BMJ. 2011;342:1404-1404.

39. Fraser D, Forman W, Fortes M, Spiva L, Hallen C. Early mobility in the intensive care unit: a community hospital's experience. Crit Care Nurse. 2015;115:49-58.

40. Harris C, Shahid S. Physical therapy-driven quality improvement to promote early mobility in the intensive care unit. Proc (Bayl Univ Med Cent). 2014;27:203-207.

41. Matthews H, Clark D, Lowman J, Sheils K. Early mobilization in trauma ICU decreases length of stay and complications. J Acute Care Phys Ther. 2010;1:72.

42. Needham DM, Korupolu R, Zanni JM, et al. Early physical medicine and rehabilitation for patients with acute respiratory failure: A quality improvement project. Arch Phys Med Rehabil. 2010;9: 536-542.

43. Talley C, Wonnacott R, Schuette J, Jamieson J, Heung M. Extending the benefits of early mobility to critically ill patients undergoing continuous renal replacement therapy: the Michigan experience. Crit Care Nurs Q. 2013;36:89-100.

44. Thompson C, Goad A, Taylor K, et al. The role of a critical care nurse in the organization and management of an early ICU mobility team. Crit Care Nurse. 2008;28:e17.

45. Winkelman C, Johnson K, Hejal R, et al. Examining the positive effects of exercise in intubated adults in ICU: a prospective repeated measures clinical study. Intensive Crit Care Nurs. 2012;28: 307-318.

46. Fitzgibbon L. Let's get moving: an interdisciplinary approach to early mobility in the medical intensive care unit. Crit Care Nurse. 2012;32: e48-e49.

47. Winkelman C, Peereboom K. Staff-perceived barriers and facilitators. Crit Care Nurse. 2010;30: S13-S16.

48. Doherty K, Yoon J, Pecanac K, Brown R, Mahoney $\mathrm{J}$. Frequency and duration of nursing care related to older patient mobility. I Nurs Scholarsh. 2014;46:20-27.

49. Garzo J, Ryan C, Waak K, et al. Early mobilization in critically ill patients: patients' mobilization level depends on health care providers profession. PMR. 2011;3:307-313.

50. Needham DM, Korupolu R. Rehabilitation quality improvement in an intensive care unit setting: Implementation of a quality improvement model. Top Stroke Rehabil. 2010;17:271-281.

51. Bailey P, Thomsen GE, Spuhler VJ, et al. Early activity is feasible and safe in respiratory failure patients. Crit Care Med. 2007;35:139-145.

52. Zanni JM, Korupolu R, Fan E, et al. Rehabilitation therapy and outcomes in acute respiratory failure: An observational pilot project. J Crit Care. 2010;25:254-262.

53. Bittner NP, Gravlin G. Critical thinking, delegation, and missed care in nursing practice. J Nurs Adm. 2009;39:142-146.

54. Doherty-King BB. How nurses decide to ambulate hospitalized older adults: development of a conceptual model. Gerontologist. 2011;51:786-797.

55. Kalisch BJ. Missed nursing care: a qualitative study. J Nurs Care Qual. 2006;21:306-313.

56. Balas M, Vasilevskis E, Burke W, et al. Critical care nurses' role in implementing the "ABCDE Bundle " into practice. Crit Care Nurse. 2012;32:35-48.

57. Hopkins R. Early activity in the ICU: Beyond safety and feasibility. Crit Care Nurse. 2010;55:481-484.

58. Engel HJ, Needham DM, Morris PE, Gropper MA. ICU early mobilization: from recommendation to implementation at three medical centers. Crit Care Med. 2013;41:S69-S80.

59. Hildreth AN, Enniss T, Martin RS, et al. Surgical intensive care unit mobility is increased after institution of a computerized mobility order set and intensive care unit mobility protocol: a prospective cohort analysis. Am Surg. 2010;76:818-822.

60. Morris PE, Herridge MS. Early intensive care unit mobility: Future directions. Crit Care Clin. 2007;23:97-110.

How to cite this article: Zhu Y-P, Xia L-X, Li G-H. Management of early mobilization in intensive care units: a multicenter cross-sectional study. Front Nurs. 2018; 4: 291-300. https://doi.org/10.1515/fon-2018-0043. 